\title{
Phonon dispersion in hypersonic two-dimensional phononic crystal membranes
}

\author{
B. Graczykowski, ${ }^{1, *}$ M. Sledzinska, ${ }^{1}$ F. Alzina, ${ }^{1}$ J. Gomis-Bresco,,${ }^{1, \dagger}$ J. S. Reparaz, ${ }^{1}$ \\ M. R. Wagner, ${ }^{1}$ and C. M. Sotomayor Torres ${ }^{1,2}$ \\ ${ }^{1}$ ICN2 - Institut Catala de Nanociencia i Nanotecnologia, Campus UAB, 08193 Bellaterra (Barcelona), Spain \\ ${ }^{2}$ ICREA - Institucio Catalana de Recerca i Estudis Avançats, 08010 Barcelona, Spain
}

(Received 13 November 2014; revised manuscript received 15 January 2015; published 13 February 2015)

\begin{abstract}
We investigate experimentally and theoretically the acoustic phonon propagation in two-dimensional phononic crystal membranes. Solid-air and solid-solid phononic crystals were made of square lattices of holes and Au pillars in and on $250 \mathrm{~nm}$ thick single crystalline Si membrane, respectively. The hypersonic phonon dispersion was investigated using Brillouin light scattering. Volume reduction (holes) or mass loading (pillars) accompanied with second-order periodicity and local resonances are shown to significantly modify the propagation of thermally activated $\mathrm{GHz}$ phonons. We use numerical modeling based on the finite element method to analyze the experimental results and determine polarization, symmetry, or three-dimensional localization of observed modes.
\end{abstract}

DOI: 10.1103/PhysRevB.91.075414

PACS number(s): 63.22.-m, 78.35.+c, 02.70.Dh

\section{INTRODUCTION}

Tuning the phonon dispersion relation, i.e., phonon engineering, provides a means of controlling related properties such as group velocity and, ultimately, phonon propagation, with relevance from sound to heat transport. Both periodic modulation of the elastic properties in phononic crystals (PnCs) [1-6] and reduction of the characteristic dimensions as in, e.g., thin membranes, thin films, and nanowires [7-10] lead to acoustic phonon propagation which is quite different than for bulk systems. The artificial, second-order periodicity introduced in PnCs results in the modification of the phonon dispersion and, optionally, in complete frequency band gaps due to Bragg reflections and/or local resonances, which can be controlled by geometry and material properties $[1,3,11,12]$. The first experimental studies on PnCs, limited by fabrication capabilities (spacing of $\mathrm{mm}$ ), were focused on sound $(\mathrm{kHz})$ and ultrasound ( $\mathrm{MHz}$ ) waves propagation and intended for applications in acoustic filtering, sensing, and wave-guiding [13-18]. Recent advances in fabrication methods have allowed reduction of the characteristic sizes of PnCs to $\mathrm{nm}$ scale, enabling the control of hypersonic $(\mathrm{GHz})$ phonons $[2,4,6,19]$ and heat transport [20,21]. Therefore, such structures have become attractive for optomechanics, radio frequency (RF), and thermoelectric applications [17,22,23]. Various one-, two-, three-dimensional PnCs designed to tune the propagation of bulk [2-4,13-15,24], surface (Rayleigh, Sezawa) [6,25-27], and plate (Lamb) waves $[5,16,18,28]$ have been investigated both theoretically and experimentally in a wide range of sizes and frequencies. However, the direct measurements of the hypersonic phonon dispersion were performed mostly for bulk [2-4,24,29,30] and surface PnCs [6,31-33], while the influence of the phononic patterning in thin membranes was studied theoretically and experimentally by means of transmission measurements, where the acoustic waves are generated

\footnotetext{
${ }^{*}$ Corresponding author: bartlomiej.graczykowski@icn.cat

${ }^{\dagger}$ Present address: ICFO-Institut de Ciencies Fotoniques and Universitat Politcnica de Catalunya, Mediterranean Technology Park, 08860 Castelldefels (Barcelona), Spain
}

and detected electrically $[5,18,34,35]$ or optically [28] in the $\mathrm{kHz}-\mathrm{MHz}$ range.

In this paper we consider two model two-dimensional (2D) PnC membranes which combine different strategies, such as spatial confinement, periodic modulation, and local resonances, affecting the dispersion of acoustic phonons. Both structures are formed on a $250 \mathrm{~nm}$ thick Si membrane, which is also used as a reference sample showing 1D confinement and phonon discretization [9,36,37]. The first sample, a solid-air PnC shown in Figs. 1(a) and 1(b), is made by removal of a periodic array of cylinders in a membrane. Here, dispersion properties are driven by the 1D confinement and Bragg scattering. The second sample, a solid-solid PnC depicted in Figs. 1(c) and 1(d), made of a square lattice of $\mathrm{Au}$ pillars on the membrane, provides an additional feature based on the local resonances. The characteristic sizes of the samples were chosen to match the appropriate range of wave numbers and spectral capabilities of the experimental setup. Here, by utilizing contactless and nondestructive Brillouin light scattering (BLS) [30,38,39] spectroscopy we show direct experimental evidence of the modification of the dispersion of $\mathrm{GHz}$ thermally activated phonons propagating in the considered PnCs. Moreover, we applied the finite element method (FEM) $[1,5,6,11,28]$ to obtain the theoretical dispersion relations and, by introducing quantitative parameters, to describe polarization, symmetry, and localization of acoustic modes. The obtained results provide novel insight regarding the $\mathrm{GHz}$ phonon dynamics in the structures potentially attractive for high-frequency applications and heat transport management.

\section{MATERIALS AND METHODS}

The PnCs shown in Fig. 1 were fabricated from siliconon-insulator (SOI) wafers with device layers of $250 \mathrm{~nm}$ purchased from Soitec Inc. and cut into $1 \times 1 \mathrm{~cm}^{2}$ chips. Poly(methyl methacrylate) (PMMA) was spun on the SOI chips and patterned by means of electron-beam litography (EBL) using a Raith 150 system. After development in a methyl isobutyl ketone:isopropanol solution (1:3) the chips were baked for $1 \mathrm{~min}$ at $70^{\circ} \mathrm{C}$. The PMMA pattern was then transferred to the SOI chip using a Bosh process with $\mathrm{SF}_{6}$ and 

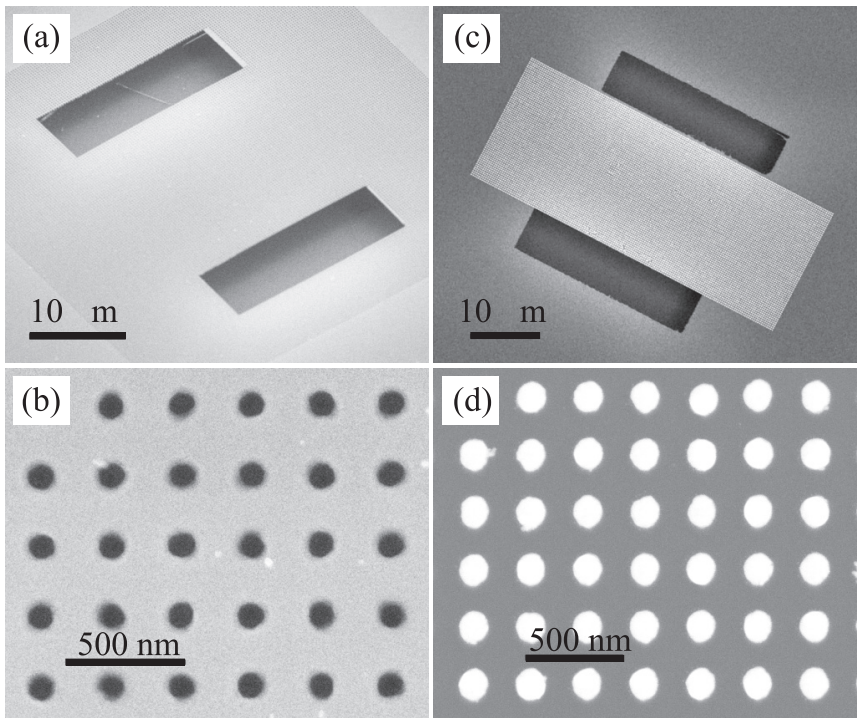

FIG. 1. Scanning electron microscope images of the studied samples. (a),(b) The solid-air PnC: holes in $250 \mathrm{~nm}$ thick $\mathrm{Si}$ membrane. (c),(d) The solid-solid PnC: Au pillars on $250 \mathrm{~nm}$ thick Si membrane.

$\mathrm{C}_{4} \mathrm{~F}_{8}$ gases with flows rates of 150 and $100 \mathrm{sccm}$, respectively (Alcatel AMS-110). The dry etching process was optimized in order to obtain vertical etching and reduce the roughness of the walls of the holes. The etching profile inside the holes was verified by imaging with a scanning electron microscope (SEM) a cross section cut by a focused ion beam (FIB). Finally the membranes were released in $49 \%$ hydrofluoric acid (HF) and dried using a critical point dryer. The typical dimensions of the resulting PnCs were of $20 \times 50 \mu \mathrm{m}$ in area, with hole diameters of $d=100 \mathrm{~nm}$ and lattice parameter of $a=300 \mathrm{~nm}$ (see Fig. 2). In addition, PnCs based on Au pillars were fabricated by EBL and evaporation of $\mathrm{Cr}$ and $\mathrm{Au}$ layers of 5 and $90 \mathrm{~nm}$, respectively. The membranes were then patterned by means of EBL and RIE and finally the structure was released by underetching with HF and dried in a critical point dryer. The relative orientation of the phononic lattices and the crystalline directions of $\mathrm{Si}$ is shown in Figs. 2(a)-2(c). For the numerical simulations the shape of the pillar was approximated by that of a thimble (truncated cone) with base diameter $d=158 \mathrm{~nm}$, semiangle $\delta=10^{\circ}$, and total height $h=95 \mathrm{~nm}$ [see Fig. 2(c)].

Brillouin light scattering measurements were performed using a six-pass tandem type Fabry-Perot interferometer (JRS Scientific Instruments) at room temperature and in a ( $p$-unpolarized) backscattering geometry [see Fig. 2(e)]. The incident light with a wavelength $\lambda_{0}=514.5 \mathrm{~nm}\left(\mathrm{Ar}^{+}\right.$laser $)$ was focused by using an Olympus $50 \times$ long working distance microscope objective with a numerical aperture of 0.45 . The laser spot had a diameter of approximately a few micrometres and the incident power was kept below $3 \mathrm{~mW}$. Here, similarly to the case of surface Brillouin light scattering in opaque and semitransparent materials and due to a small scattering volume, the dominant component of the inelastically scattered light comes from the surface ripple mechanism. Thus, the momentum conservation is satisfied only for the in-plane components, and the magnitude of the scattering wave vector
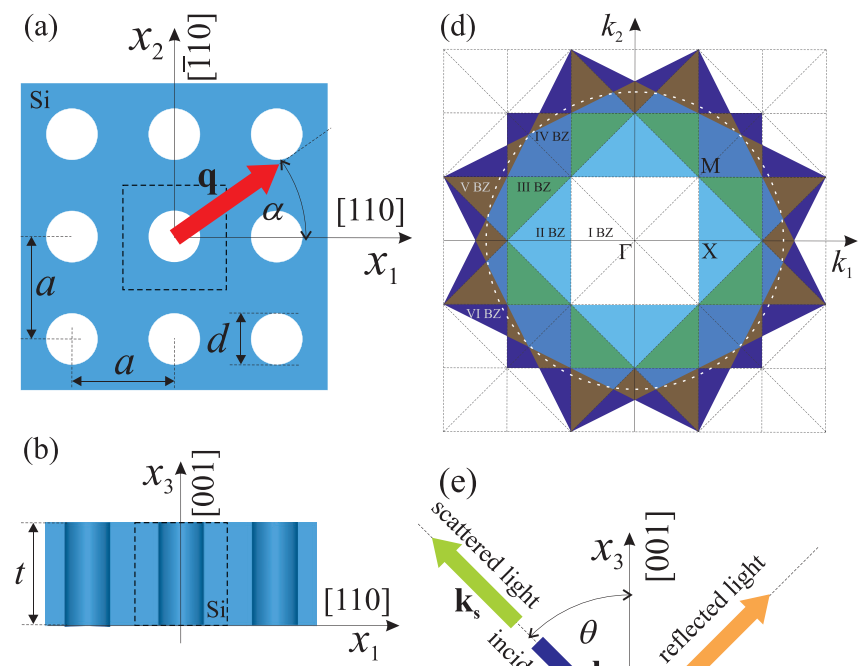

(e)
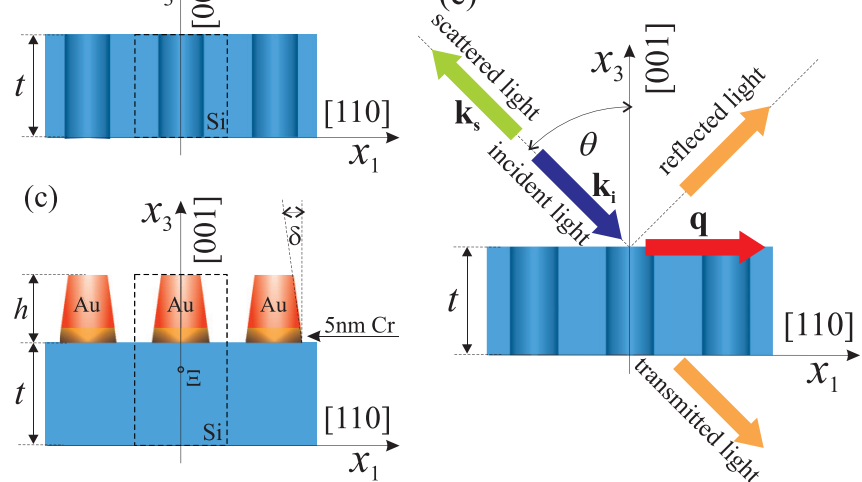

FIG. 2. (Color online) Schematic (a) top view and [(b) and (c)] lateral views showing sample orientation and characteristic sizes; (d) corresponding reciprocal lattice and range of wave numbers available in the experiment (dashed white circle); (e) lateral view, Brillouin light scattering geometry. Symbols $\mathbf{k}_{\mathbf{i}}, \mathbf{k}_{\mathbf{s}}$, and $\mathbf{q}$ denote incident light, scattered light, and scattering wave vectors respectively, and $\theta$ is the scattering angle.

$\mathbf{q}$ is as follows: [38,39]:

$$
q=\frac{4 \pi \sin \theta}{\lambda_{0}},
$$

where $\theta$ is the incident and scattered light angle. Therefore, by changing $\theta$ the wave number $q$ can be adjusted and the phonon dispersion can be determined. Since the wavelength $\lambda_{0}$ is fixed, Eq. (1) also defines the range of wave numbers available experimentally. Potentially measurable scattering wave vectors and Brillouin zones are enclosed by the dashed circle in Fig. 2(d). In our case $q$ can be varied from 0 to (approximately) $0.0244 \mathrm{~nm}^{-1}$ and, thus, we can measure the first to fourth Brillouin zones of the fabricated PnCs.

\section{RESULTS AND DISCUSSION}

\section{A. Thin Si membrane}

The breaking of a spatial continuity in one dimension together with stress-free boundary conditions results in the propagation of three families of well-known Lamb waves: antisymmetric (A), symmetric (S), and shear horizontal (SH) $[36,37]$. In the general case of anisotropic materials the phonon dispersion for relatively small wave numbers can be satisfactorily determined by numerical calculations based on the elastic continuum theory $[9,41]$. Assuming the longwavelength approximation, membranes without phononic 

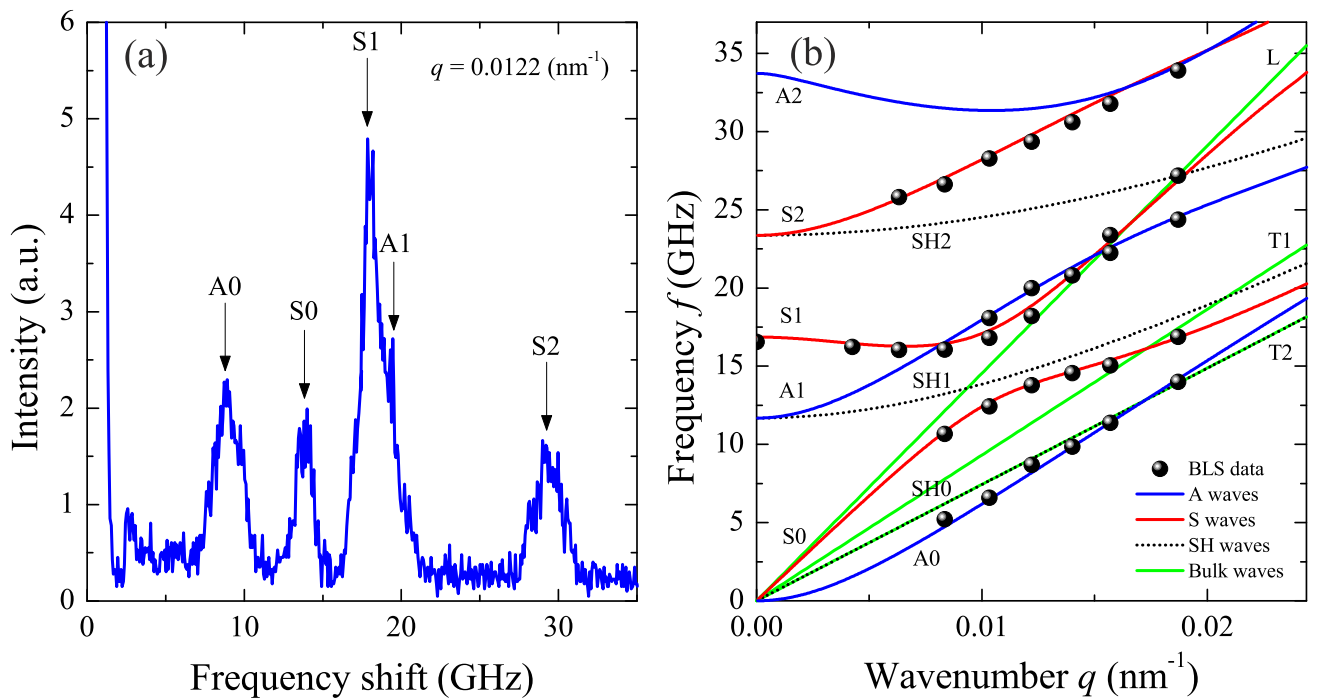

FIG. 3. (Color online) (a) BLS spectrum (anti-Stokes) at $q=0.0122 \mathrm{~nm}^{-1}$ and (b) measured and calculated phonon dispersion relation obtained for the [110] (001) of the $250 \mathrm{~nm}$ thick Si membrane (substrate). Symbols L, T1, and T2 stand for longitudinal and fast and slow transverse acoustic waves propagating in bulk $\mathrm{Si}$, respectively.

structures are effectively homogenous, thus the wave vector $\mathbf{q}$ and the wave vector $\mathbf{k}$ of the acoustic phonon are equal.

Figure 3(a) displays a particular BLS spectrum (anti-Stokes component) obtained at $q=0.0122 \mathrm{~nm}^{-1}$ for a $250 \mathrm{~nm}$ thick membrane without any phononic structure. This sample was measured as a reference for the BLS experiments performed on PnCs based on the membranes with the same thickness and elastic properties. The position of a given peak, obtained by the Lorentzian fit, indicates the frequency of the corresponding acoustic phonon with the wave number $q$. Here, the BLS spectrum shows five peaks, which are related to the $\mathrm{A}$ and $\mathrm{S}$ families of waves: fundamentals (A0 and S0) and higher harmonics (A1, S1, S2). The height of a particular peak is determined by the intensity of the inelastically scattered light, which depends on the mean-squared amplitude of the out-of-plane displacement, scattering geometry, and optical setup $[38,39]$. Figure 3(b) depicts the results of the angle resolved BLS compared to the theoretical calculations. The measured frequencies of symmetric and antisymmetric modes are in good agreement with predictions of the classical elastodynamics. For all the SH modes the out-of-plane displacement is zero, therefore they do not contribute to the ripple mechanism and they are not present in the BLS spectrum. The dispersion curve of Fig. 3(b) is scalable by the product of both frequency and wave vector and the plate thickness, thus is applicable to any range of frequencies in the long-wavelength limit.

\section{B. Solid-air phononic crystal membrane}

For the BLS measurements of the PnCs the scattering wave vector is defined by momentum conservation $\mathbf{q}=\mathbf{k}+\mathbf{G}$, where $\mathbf{G}=(2 \pi m / a, 2 \pi n / a)$ denotes the reciprocal lattice vector and $n, m$ are integers. As follows from Fig. 2(a) both square arrays of holes and pillars hold fourfold rotational symmetry with respect to the $x_{3}$ axis. Thus, the irreducible Brillouin zone of PnCs is enclosed by the contour $Г Х M \Gamma$ as shown in Fig. 2(d). In order to obtain the phonon dispersion relation of the PnCs, FEM calculations were performed taking the unit cell indicated by a dashed line in Figs. 2(a)-2(c), applying periodic Bloch boundary conditions for the boundaries normal to $x_{1}$ and $x_{2}$ axes and free boundary conditions for planes normal to the $x_{3}$ axis $[3,4,11,41]$. As it follows from Fig. 2(a), axes $x_{1}$ and $x_{2}$ lie along [110] and [110], respectively. This implies the rotation of the elastic constants $C_{I J}$ of silicon, which for the initial system $\left(x_{1} \|[100]\right.$ and $\left.x_{2} \|[010]\right)$ are given by three nonzero independent values: $C_{11}, C_{12}$, and $C_{44}$. After rotation of $45^{\circ}$ around the $x_{3}$ axis the new elastic constants $C_{I J}^{\prime}$ are $C_{11}^{\prime}=C_{22}^{\prime}=\left(C_{11}+C_{12}+2 C_{44}\right) / 2, C_{33}^{\prime}=$ $C_{11}, C_{44}^{\prime}=C_{55}^{\prime}=C_{44}, C_{66}^{\prime}=\left(C_{11}-C_{12}\right) / 2, C_{12}^{\prime}=\left(C_{11}+\right.$ $\left.C_{12}-2 C_{44}\right) / 2$, and $C_{13}^{\prime}=C_{23}^{\prime}=C_{12}$. The elastic constants of $\mathrm{Au}$ (pillars) and $\mathrm{Cr}$ (5 $\mathrm{nm}$ adhesion layer) are assumed to be isotropic, therefore together with the mass density (scalar) they remain unchanged after the rotation. All the material properties (initial) for $\mathrm{Si}, \mathrm{Au}$, and $\mathrm{Cr}$ used in calculations are gathered in Table I. Figure 4 displays two particular BLS spectra obtained for $\Gamma X$ and $\Gamma M$ directions of the $\mathrm{PnC}$ made of air holes in the Si membrane [see Figs. 1(a) and 1(b)]. These results exhibit quite different spectral features in comparison to the spectra of the reference sample [Fig. 3(a)]. In Fig. 4 the particular peaks are identified as coming from the corresponding acoustic modes by means of the FEM displacement fields. Similarly as for the reference sample, only the modes with a dominant out-of-plane displacement component are visible in the BLS spectra. BLS results obtained for several different scattering angles for $\Gamma X$ and $\Gamma M$ directions are successfully compared to the FEM calculations in the form of the dispersion relation in

TABLE I. Mass density $\rho$ and elastic constants $C_{I J}$ of $\mathrm{Si}, \mathrm{Au}$, and $\mathrm{Cr}$ used in FEM calculations [40].

\begin{tabular}{lcccc}
\hline \hline & $\rho\left(\mathrm{kg} / \mathrm{m}^{3}\right)$ & $C_{11}(\mathrm{GPa})$ & $C_{12}(\mathrm{GPa})$ & $C_{44}(\mathrm{GPa})$ \\
\hline $\mathrm{Si}$ & 2331 & 165.7 & 63.9 & 79.9 \\
$\mathrm{Au}$ & 19300 & 226.9 & 178.2 & 24.3 \\
$\mathrm{Cr}$ & 7150 & 314.1 & 83.5 & 115.3 \\
\hline \hline
\end{tabular}



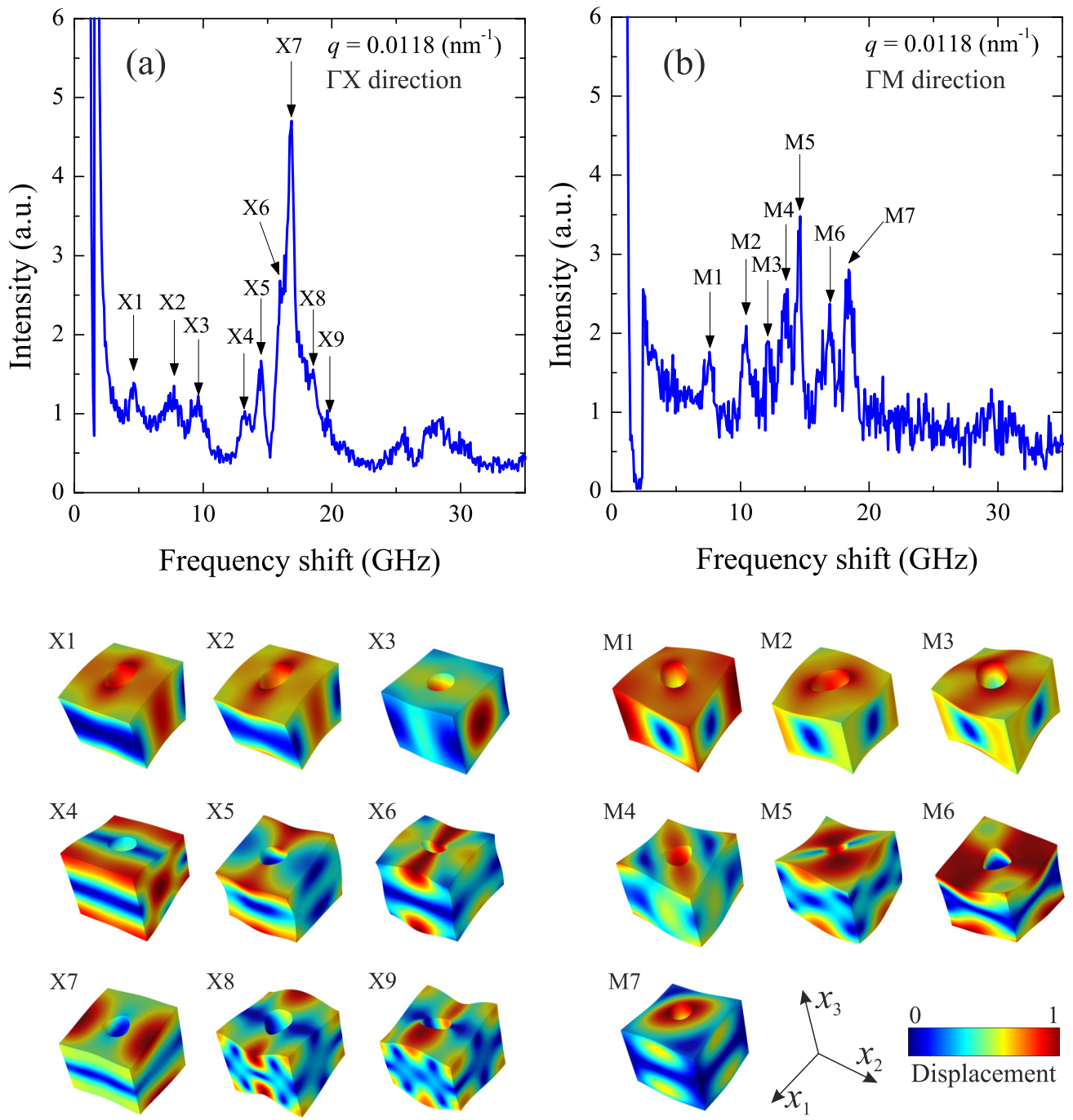

FIG. 4. (Color online) BLS spectra (anti-Stokes) and corresponding FEM deformation fields of the solid-air PnC obtained for (a) $\Gamma X$ direction at $q=0.0118 \mathrm{~nm}^{-1}$ and (b) $\Gamma M$ direction at $q=0.0118 \mathrm{~nm}^{-1}$.

Figs. 5(a) and 5(b), respectively. It is clear that the square lattice of air holes in the $\mathrm{Si}$ membrane introduces band folding and splitting when the Bragg condition is satisfied as well as anticrossing between related branches, and results in phonon dispersion completely different from that of the reference sample [Fig. 3(b)]. Here, according to the Bloch theorem frequency $f$ of acoustic modes satisfy the relation: $f(\mathbf{k})=f(\mathbf{k}+\mathbf{G})$. Figure 5(a) shows pseudoband gap for symmetric modes (12.23-13.26 GHz) and small full band gap (12.81-13.16 GHz) along $\Gamma X$. For both measured directions, modes with frequencies higher than $20 \mathrm{GHz}$ were difficult to identify due to the numerous experimental peaks and FEM solutions, so closely packed that they form a continuum of modes in Figs. 4 and 5. As it was shown for similar PnCs, a full acoustic band gap, desirable for potential applications, can be reached simply by increasing the hole diameter $[20,35]$. Furthermore, the central frequency of the acoustic band gap can be adjusted by rescaling the $\mathrm{PnC}$ characteristic sizes, i.e., keeping the thickness to diameter ratio fixed. The separation of modes into A and S types shown in Fig. 5(c) is only possible for the solid-air PnCs since pillars break the mirror symmetry of $x_{1} x_{2}$ plane at $x_{3}=t / 2$. The symmetry with respect to the membrane mid-plane of a particular mode can be determined by comparing corresponding components $u_{i}(i=\{1,2,3\})$ of the displacement at $x_{3}=0$ and $x_{3}=t$ :

$$
\chi_{i}=\frac{1}{S} \int_{S} \frac{\left.u_{i}\right|_{x_{3}=0}}{\left.u_{i}\right|_{x_{3}=t}} d S .
$$

In the equation above all the corresponding points $\left(x_{1}, x_{2}\right.$, $\left.x_{3}=0\right)$ and $\left(x_{1}, x_{2}, x_{3}=t\right)$ are compared, then the integral over the area $S$ is normalized. As a result the symmetric modes [red lines in Fig. 5(c)] are given by $\chi_{i}=(1,1,-1)$ and the antisymmetric modes [blue lines in Fig. 5(c)] by $\chi_{i}=(-1,1,1)$. No other sets of the parameter $\chi_{i}$ appeared in the FEM calculations, which means that mixing of symmetric and antisymmetric in-plane and out-of-plane components of displacement is forbidden. Figure 4 show displacement fields of antisymmetric $(X 1, X 2, X 3, M 1, M 2, M 7)$ and symmetric $(X 3, X 4, X 5, X 7, X 8, X 9, M 3, M 4, M 5, M 6)$ modes. For both types of the considered PnCs the polarization of the acoustic modes can be analyzed applying FEM postprocessing calculations. Here, we define components of the 

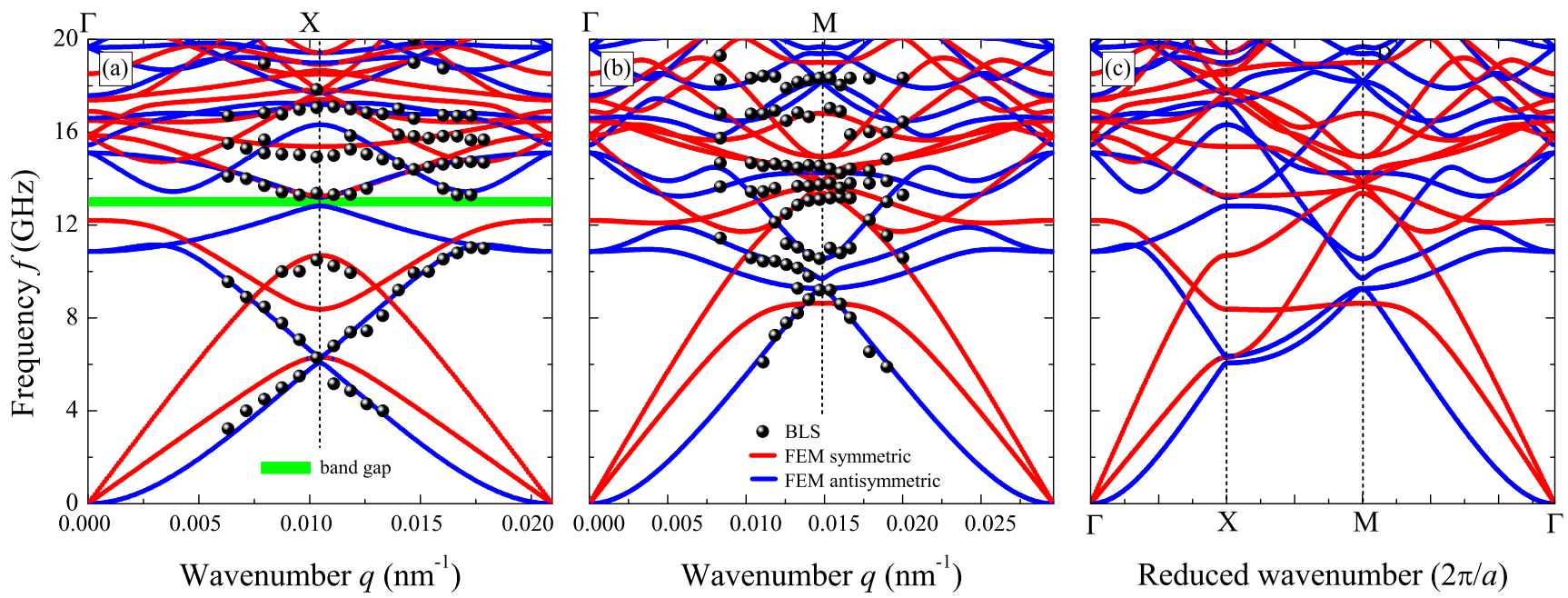

FIG. 5. (Color online) Measured (spheres) and calculated (lines) phonon dispersion of the solid-air PnC. (a) $\Gamma X$ direction, (b) $\Gamma M$ direction, and (c) reduced IBZ showing symmetry and antisymmetry of modes according to Eq. (2).

total displacement: $P_{1}$ - parallel to $\mathbf{q}, P_{2}$ - perpendicular to $\mathbf{q}$ and lying in the $x_{1} x_{2}$ plane, $P_{3}$ - perpendicular to $\mathbf{q}$ and parallel to the $x_{3}$ axis. All these components can be determined from the equation

$$
P_{j}=\frac{1}{V} \int_{V} \frac{\left|u_{i} r_{i j}\right|}{\left(u_{k} u_{k}\right)^{1 / 2}} d V,
$$

where the summation convention is used $(i, j, k,=\{1,2,3\})$, $u_{i}$ stands for the component of displacement in the $x_{i}$ axis, and $V$ is the volume of the unit cell. $r_{i j}$ denotes components of the rotation matrix, which are defined by the angle $\alpha$ between the scattering vector $\mathbf{q}$ and $x_{1}$ axis [see Fig. 2 (a)]. The values of three possible components $P_{j}$ of the total displacement are presented in Fig. 6 as a color scale in the dispersion relation. Here, we can find that the polarization for all modes is mixed and depends on the wave number, direction, and particular branch. ContrarilyIn contrast to the case of the plane membrane, modes at the $\Gamma$ point cannot be separated into pure longitudinal or transverse waves. Moreover, $P_{2}$, describing the in-plane displacement, can take values ranging from 0 to 1 for all the wave numbers in the 1st Brillouin Zone (I BZ). This implies that $\mathrm{A}$ and $\mathrm{S}$ modes can have a nonzero in-plane and normal-to-q component of the displacement. Consequently, Fig. 5(c) depicts only the separation into A and $\mathrm{S}$ modes. Parameter $P_{j}$ for a given branch of the dispersion relation shows continuous behavior with respect to the wave number. Therefore, it gives a possibility to sort FEM solutions, what is crucial for, e.g., group velocity and density of states calculations.

\section{Solid-solid phononic crystal membrane}

A similar approach, based on BLS and FEM, was applied to the solid-solid PnC made of a square array of $\mathrm{Au}$ pillars on a Si membrane [see Figs. 1(c) and 1(d)]. Figure 7 shows two particular BLS spectra obtained for $\Gamma X$ and $\Gamma M$ directions at $q=0.0084 \mathrm{~nm}^{-1}$ and $q=0.0122 \mathrm{~nm}^{-1}$, respectively. As follows from Fig. 7, peaks labeled $X 1$ and $M 1$ are sharp
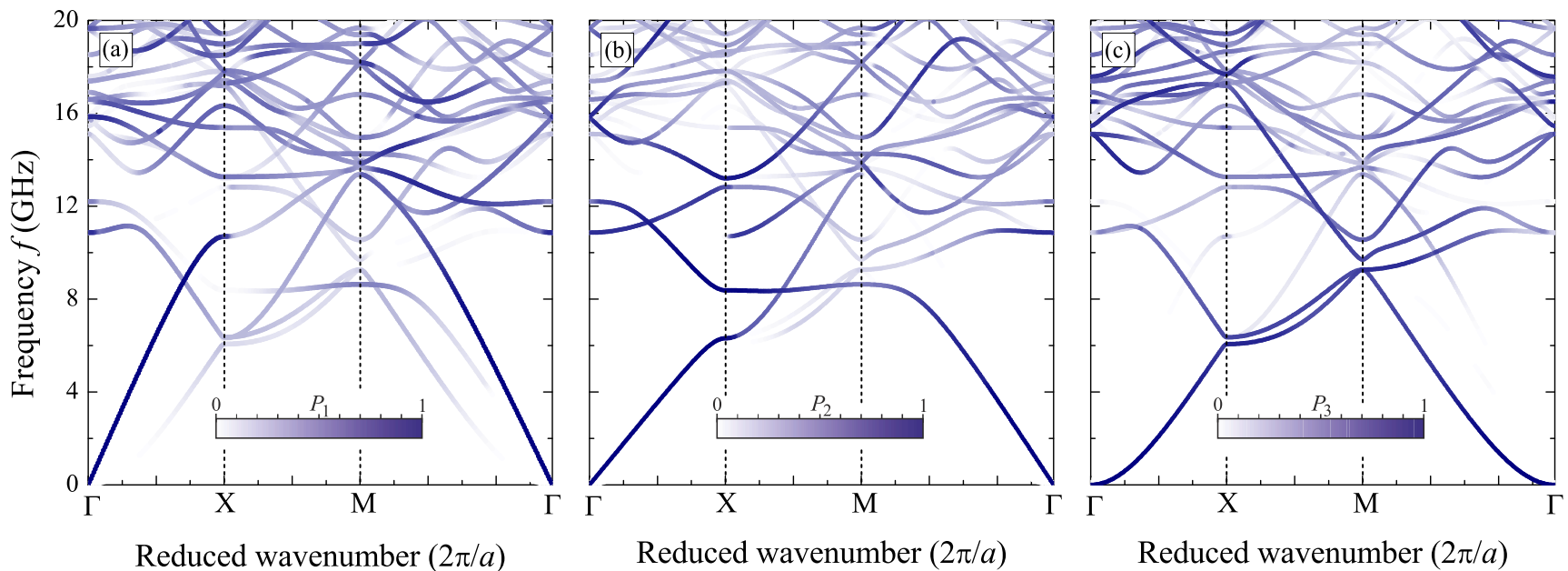

FIG. 6. (Color online) Calculated phonon dispersion of $\mathrm{PnC}$ made of air holes in the Si membrane showing the contribution of (a) $P_{1}$, (b) $P_{2}$, and (c) $P_{3}$ components to the total displacement according to Eq. (3). 

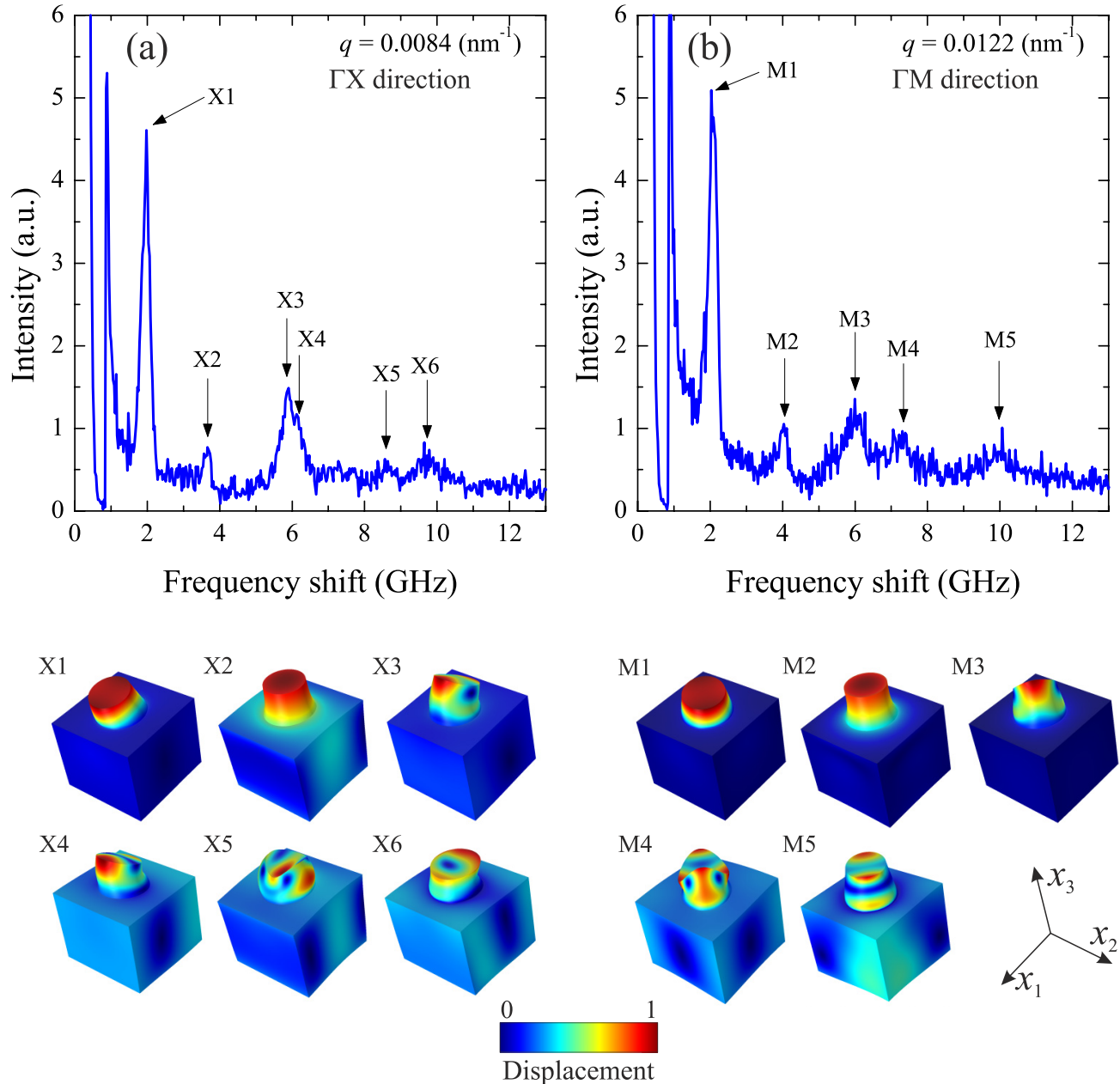

Displacement

FIG. 7. (Color online) BLS spectra (anti-Stokes) and corresponding FEM deformation fields of the solid-solid PnC obtained for (a) $\Gamma X$ direction at $q=0.0084 \mathrm{~nm}^{-1}$ and (b) $\Gamma M$ direction at $q=0.0122 \mathrm{~nm}^{-1}$.

and relatively high. This can be explained if we consider the corresponding FEM displacement fields. Modes $X 1$ and $M 1$ are mainly localized in the pillar with a small deformation of the membrane. Similar spectral features showing localized modes due to the presence of the local resonances were reported for surface $\mathrm{PnCs}[6,31,32]$. In the case of local resonances in solid-solid PnCs made of pillars it is useful to introduce a parameter, which indicates the center [point $\Xi$
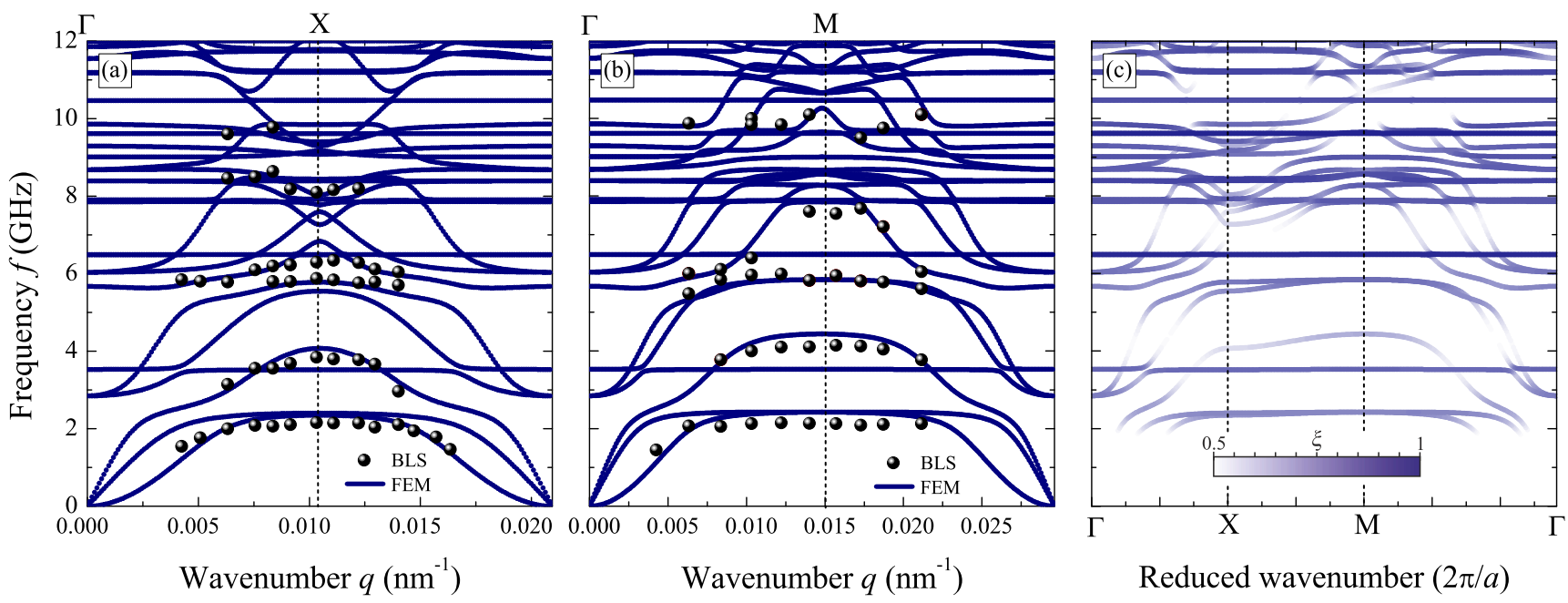

FIG. 8. (Color online) Measured (spheres) and calculated (lines) phonon dispersion of the solid-solid PnC. (a) $\Gamma X$ direction, (b) $\Gamma M$ direction, and (c) reduced IBZ showing the relative position of the elastic energy center in the $x_{3}$ axis according to Eq. (4). 

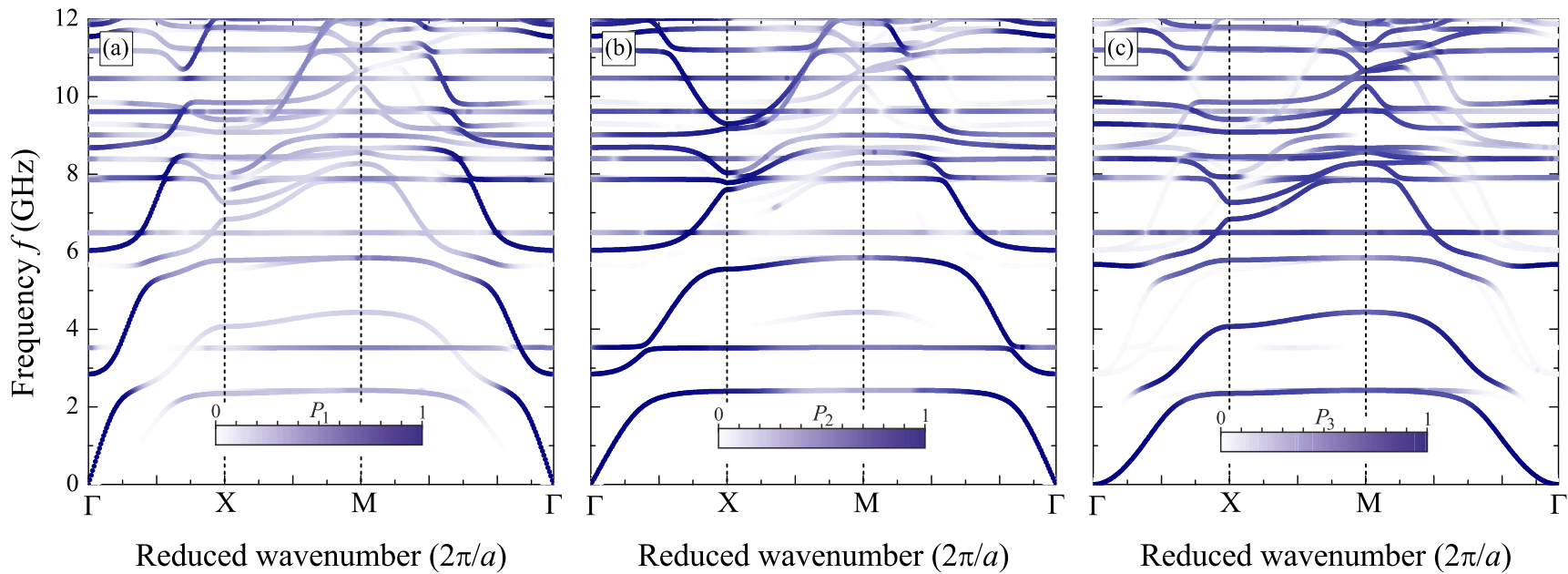

FIG. 9. (Color online) Calculated phonon dispersion of the solid-solid PnC showing the contribution of (a) $P_{1}$, (b) $P_{2}$, and (c) $P_{3}$ components to the total displacement according to Eq. (3).

in Fig. 2(c)] of the elastic energy in the $x_{3}$ axis

$$
\xi=\left(\frac{1}{t+h}\right) \frac{\int_{V} F x_{3} d V}{\int_{V} F d V}
$$

where integrals are taken over a whole volume of the unit cell, and $F=C_{i j k l} u_{i j} u_{k l}$ denotes the free elastic energy density $[6,42]$. Since $\xi$ is normalized to the total height of the unit cell [see Fig. 2 (c)], in our case, modes with $\xi>0.71$ are practically localized in the pillars. The exemplary displacement fields of the modes showing this 3D confinement of the elastic energy $(X 1, X 3, M 1, M 2, M 3)$ are depicted in Fig. 7. The experimentally measured dispersion relation is compared with the FEM calculations in Figs. 8(a) and 8(b). The agreement is quite satisfactory, however with a greater deviation than that found for the solid-air PnC [see Figs. 5(a) and 5(b)]. This difference between theory and experiment can be related to the fabrication process of the samples. It is easier to keep the same diameter of holes than to hold all the characteristic sizes of pillars, which determine frequencies of the local resonances $[6,11,43]$. Nevertheless, it is clear that loading of the membrane with the periodic array of relatively heavy $\mathrm{Au}$ pillars results in a strong modification of the phonon dispersion with an overall frequency downshift of the branches with respect to the plane membrane. Moreover, the hybridization between local resonances in pillars and modes of the underlying membrane results in an anticrossing between the nondispersive branches of the local resonances and the bands of the propagating modes in the substrate. This may lead to the opening of band gaps not related to Bragg reflections, and at frequencies not reachable by the latter, which can be tuned by changing the characteristic sizes of the pillars [43,44]. As mentioned above, pillars break the mirror symmetry and FEM solutions cannot be separated into A and S types. The introduced parameter $\xi$, the values of which are shown in Fig. 8(c), clearly correlates with the horizontal branches of the localized states of pillars. Figure 9 depicts the contribution of normal components $P_{j}$ to the total displacement. Similarly as for the solid-air PnCs, all the modes show the mixed polarization. Moreover, from Figs. 8(c) and 9(c) we can find that waves measurable using BLS are those of strong 3D localisation and a dominant out-of plane component of the displacement $P_{3}$.

\section{CONCLUSIONS}

Solid-air and solid-solid 2D phononic crystals made of $\mathrm{Si}$ membranes with a thickness of $250 \mathrm{~nm}$ and comparable characteristic sizes of hundreds of nanometres were investigated. The dispersion relation of thermally activated $\mathrm{GHz}$ phonons was measured directly by means of Brillouin light scattering and compared with numerical calculations based on the finite element method. The measured phononic crystals show significant changes in the acoustic phonon propagation due to the Bragg scattering (both samples) and the presence of the local resonances (pillars on $\mathrm{Si}$ ) with respect to the $\mathrm{Si}$ membrane (substrate). We applied an extended FEM study introducing the parameters, which can determine symmetry and quantify localization and polarization of the acoustic modes in the considered PnCs. The solid-air PnC holds the mid-plane mirror symmetry of the displacement. However, in contrast to the plane membrane, all the modes show mixed polarization, where in-plane and out-of-plane modes do not propagate separately. The solid-solid PnC, besides the mixed polarization of modes, shows a hybridization of local resonances with propagating modes of the substrate. The 3D confinement of the acoustic modes in pillars was observed experimentally as very pronounced peaks with frequencies almost independent of the wave number and direction of propagation. The presented results have deep implications for engineering of the hypersound and heat transport in the nanoscale phononic structures and metamaterials.

\section{ACKNOWLEDGMENTS}

The authors acknowledge financial support from the European FP7 project MERGING (Grant No. 309150), the Spanish MINECO projects nanoTHERM (Grant No. CSD2010- 
0044) and TAPHOR (MAT2012-31392) and the program Severo Ochoa (Grant SEV-2013-0295). M.R.W. acknowledges support of the Marie Curie Fellowship HeatProNano (Grant No. 628197).
[1] Y. Pennec, J. O. Vasseur, B. Djafari-Rouhani, L. Dobrzynski, and P. A. Deymier, Surf. Sci. Rep. 65, 229 (2010).

[2] T. Gorishnyy, J.-H. Jang, C. Koh, and E. L. Thomas, Appl. Phys. Lett. 91, 121915 (2007).

[3] T. Still, W. Cheng, M. Retsch, R. Sainidou, J. Wang, U. Jonas, N. Stefanou, and G. Fytas, Phys. Rev. Lett. 100, 194301 (2008).

[4] D. Schneider, F. Liaqat, E. H. El Boudouti, Y. El Hassouani, B. Djafari-Rouhani, W. Tremel, H.-J. Butt, and G. Fytas, Nano Lett. 12, 3101 (2012).

[5] A. Khelif, B. Aoubiza, S. Mohammadi, A. Adibi, and V. Laude, Phys. Rev. E 74, 046610 (2006).

[6] B. Graczykowski, M. Sledzinska, N. Kehagias, F. Alzina, J. S. Reparaz, and C. M. Sotomayor Torres, Appl. Phys. Letters 104, 123108 (2014).

[7] C. M. Sotomayor Torres, A. Zwick, F. Poinsotte, J. Groenen, M. Prunnila, J. Ahopelto, A. Mlayah, and V. Paillard, Phys. Status Solidi C 1, 2609 (2004).

[8] J. Groenen, F. Poinsotte, A. Zwick, C. M. Sotomayor Torres, M. Prunnila, and J. Ahopelto, Phys. Rev. B 77, 045420 (2008).

[9] J. Cuffe, E. Chávez, A. Shchepetov, P.-O. Chapuis, E. H. El Boudouti, F. Alzina, T. Kehoe, J. Gomis-Bresco, D. Dudek, Y. Pennec, B. Djafari-Rouhani, M. Prunnila, J. Ahopelto, and C. M. Sotomayor Torres, Nano Lett. 12, 3569 (2012).

[10] V. A. Fonoberov and A. A. Balandin, Nano Lett. 5, 1920 (2005).

[11] A. Khelif, Y. Achaoui, S. Benchabane, V. Laude, and B. Aoubiza, Phys. Rev. B 81, 214303 (2010).

[12] Y. Achaoui, A. Khelif, S. Benchabane, L. Robert, and V. Laude, Phys. Rev. B 83, 104201 (2011).

[13] F. R. Montero de Espinosa, E. Jiménez, and M. Torres, Phys. Rev. Lett. 80, 1208 (1998).

[14] Z. Liu, X. Zhang, Y. Mao, Y. Y. Zhu, Z. Yang, C. T. Chan, and P. Sheng, Science 289, 1734 (2000).

[15] J. O. Vasseur, P. A. Deymier, B. Chenni, B. Djafari-Rouhani, L. Dobrzynski, and D. Prevost, Phys. Rev. Lett. 86, 3012 (2001).

[16] X. Zhang, T. Jackson, E. Lafond, P. Deymier, and J. Vasseur, Appl. Phys. Lett. 88, 041911 (2006).

[17] R. H. Olsson III and I. El-Kady, Meas. Sci. Technol. 20, 012002 (2009).

[18] H. Estrada, F. J. Garcia de Abajo, P. Candelas, A. Uris, F. Belmar, and F. Meseguer, Phys. Rev. Lett. 102, 144301 (2009).

[19] Y. M. Soliman, M. F. Su, Z. C. Leseman, C. M. Reinke, I. El-Kady, and R. H. Olsson, Appl. Phys. Lett. 97, 193502 (2010).

[20] N. Zen, T. A. Puurtinen, T. J. Isotalo, S. Chaudhuri, and I. J. Maasilta, Nat. Commun 5, 3435 (2014).

[21] P. E. Hopkins, C. M. Reinke, M. F. Su, R. H. Olsson, E. A. Shaner, Z. C. Leseman, J. R. Serrano, L. M. Phinney, and I. El-Kady, Nano Lett. 11, 107 (2011).
[22] M. Eichenfield, J. Chan, R. M. Camacho, K. J. Vahala, and O. Painter, Nature (London) 462, 78 (2009).

[23] M. Maldovan, Nature (London) 503, 209 (2013).

[24] N. Gomopoulos, D. Maschke, C. Y. Koh, E. L. Thomas, W. Tremel, H.-J. Butt, and G. Fytas, Nano Lett. 10, 980 (2010).

[25] L. Dhar and J. A. Rogers, Appl. Phys. Lett. 77, 1402 (2000).

[26] Y. Tanaka and S.-i. Tamura, Phys. Rev. B 58, 7958 (1998).

[27] A. A. Maznev, Phys. Rev. B 78, 155323 (2008).

[28] T.-T. Wu, Z.-G. Huang, T.-C. Tsai, and T.-C. Wu, Appl. Phys. Lett. 93, 111902 (2008).

[29] A. Sato, Y. Pennec, T. Yanagishita, H. Masuda, W. Knoll, B. Djafari-Rouhani, and G. Fytas, New J. Phys. 14, 113032 (2012).

[30] L. C. Parsons and G. T. Andrews, J. Appl. Phys. 116, 033510 (2014).

[31] S. Mielcarek, A. Trzaskowska, B. Graczykowski, and J. Sarkar, Phys. Status Solidi Rapid Res. Lett. 6, 175 (2012).

[32] A. Trzaskowska, S. Mielcarek, and J. Sarkar, J. Appl. Phys. 114, 134304 (2013).

[33] C. G. Hou, V. L. Zhang, S. C. Ng, M. H. Kuok, H. S. Lim, X. M. Liu, and A. O. Adeyeye, Appl. Phys. Lett. 104, 093108 (2014).

[34] S. Mohammadi, A. A. Eftekhar, A. Khelif, W. D. Hunt, and A. Adibi, Appl. Phys. Lett. 92, 221905 (2008).

[35] M. Gorisse, S. Benchabane, G. Teissier, C. Billard, A. Reinhardt, V. Laude, E. Defa, and M. Ad, Appl. Phys. Lett. 98, 234103 (2011).

[36] H. Lamb, Proc. R. Soc. London A 93, 114 (1917).

[37] B. A. Auld, Acoustic Fields and Waves in Solids, Vol. 2 (Krieger Publishing, USA, 1990).

[38] J. D. Comins, Handbook of Elastic Properties of Solids, Liquids and Gases, edited by A. Every and W. Sachse, Vol. 1 (Academic Press, San Diego, 2001), pp. 349-378.

[39] J. Sandercock, in Light Scattering in Solids III, Topics in Applied Physics, Vol. 51, edited by M. Cardona and G. Gntherodt (Springer, Berlin, 1982), pp. 173-206.

[40] G. W. Farnell, Properties of Elastic Surface Waves, edited by W. P. Mason and R. N. Thurston, Physical Acoustics, Vol. 6 (Academic Press, New York, 1970), pp. 109-166.

[41] B. Graczykowski, J. Gomis-Bresco, F. Alzina, J. S. Reparaz, A. Shchepetov, M. Prunnila, J. Ahopelto, and C. M. Sotomayor Torres, N. J. Phys. 16, 073024 (2014).

[42] L. D. Landau and E. M. Lifshitz, Theory of Elasticity (Pergamon Press, New York, 1959).

[43] Y. Pennec, B. Djafari-Rouhani, H. Larabi, J. O. Vasseur, and A. C. Hladky-Hennion, Phys. Rev. B 78, 104105 (2008).

[44] B. L. Davis and M. I. Hussein, Phys. Rev. Lett. 112, 055505 (2014). 